\title{
Effect of nonionic surfactant and acidity on chitosan nanofibers with different molecular weights
}

\author{
Khalid Ziani ${ }^{\mathrm{a}, *}$, Catherine Henrist ${ }^{\mathrm{b}}$, Christine Jérôme $^{\mathrm{c}}$, Abdelhafid Aqil $^{\mathrm{c}}$, Juan I. Matéd, Rudi Cloots ${ }^{\mathrm{b}}$ \\ a Department of Food Science, Biopolymers and Colloids Research Laboratory, University of Massachusetts, Amherst, MA 01003, USA \\ ${ }^{\mathrm{b}}$ Inorganic Materials Chemistry (LCIS-GreenMAT), Department of Chemistry, University of Liège, Sart-Tilman B6, B-4000 Liège, Belgium \\ ${ }^{\mathrm{c}}$ Center for Education and Research on Macromolecules (CERM), University of Liège, Sart-Tilman B6a, B-4000 Liège, Belgium \\ d Departamento de Tecnología de Alimentos, Universidad Pública de Navarra, Campus Arrosadia, Pamplona, Spain
}

\section{A R T I C L E I N F O}

\section{Article history:}

Received 13 April 2010

Received in revised form 7 July 2010

Accepted 4 August 2010

Available online $\mathrm{xxx}$

\section{Keywords:}

Chitosan

Nanofibers

Molecular weight

Surfactant

\begin{abstract}
A B S T R A C T
Blend solutions of chitosan (Cs) and poly(ethylene oxide) (PEO) at different acidities were electrospun to produce membranes of nanofibers with different diameters. The use of polysorbate surfactant Tween 20 to improve functionality of these nanofibers was studied. The procedure was as follows: $5 \%(\mathrm{w} / \mathrm{v})$ of two high deacetylation degree $\mathrm{Cs}$ with different molecular weights $(\mathrm{Mw}=148 \mathrm{kDa}$ and $68 \mathrm{kDa})$ were dissolved in solutions with different acetic acid concentrations (10\%, 50\% and $90 \%(\mathrm{v} / \mathrm{v})$ ) then mixed with PEO. The influence of molecular weight and acidity on the morphology and physicochemical properties of solutions and formed nanofibers was also investigated. Results revealed that pure Cs dissolved in different acidities did not form fibers and instead it deposited as beads. Addition of PEO was necessary to electrospin all Cs solutions. Average fiber diameters and size distribution differ with acidity and molecular weight. Composite solutions of Cs, synthetic polymer PEO, and surfactant can be effectively electrospun. The presence of surfactant resulted in decrease of surface tension and in the formation of smooth or beaded fibers. This may be important for new applications since active nanofibers could serve as carriers of components such as drugs or additives.
\end{abstract}

(C) 2010 Elsevier Ltd. All rights reserved.

\section{Introduction}

Electrospinning is a successful and inexpensive technique used to produce nanofibers. In the last 10 years, nanofibers have generated a lot of interest because the use of their nanoscale properties is very promising. Electrospinning consists of applying an electric field using a high voltage source between the tip of a syringe and a collector plate, with a polymer solution being contained within the syringe. The voltage causes a jet of polymer solution to be expelled from the syringe and moved towards an appropriate collector plate. As the solvent in the jet dries, the remaining polymer solidifies and forms ultrafine nanofibers that are collected on the collector plate (Kriegel, Kit, McClements, \& Weiss, 2009a).

Researchers have focused on the application of nanofibers in various fields. Membranes based on nanofibers that have generally varying diameters from several $\mathrm{nm}$ to greater than $5 \mu \mathrm{m}$ (Quynh, Sharma, \& Mikos, 2006) can be used as drug holders, as filtration membranes, catalytic nanofibers, fiber-based sensors, and tissue engineering scaffolds (Shalumon et al., 2009). There is a wide range of spinnable polymers; however, only few of

\footnotetext{
* Corresponding author. Tel.: +1 6179341981.

E-mail address: kziani@foodsci.umass.edu (K. Ziani).
}

them have the required properties to make them suitable for use in such critical applications. In the biomedical sector, natural polymers are generally favored over synthetic polymers in view of their proven compatibility and resorbable biodegradation (Bhattarai, Edmondson, Veiseh, Matsen, \& Zhang, 2005). One of the most interesting biopolymers that gained a great attention is Cs [ $\beta$-(1-4)-2-amino-2-deoxy-D-glucopyranose]. Cs is a biomaterial obtained from alkaline deacetylated form of natural chitin, the second most abundant polysaccharide on the earth after cellulose (Kafetzopoulos, Martinou, \& Bouriotis, 1993; Zentz et al., 2001). As a biopolymer, it is considered as a promising material to produce active nanofibers with numerous properties and applications.

To facilitate Cs electrospinning, it was necessary to blend or crosslink it using other polymers such as PEO (Du \& Hsieh, 2007; Kriegel et al., 2009a) or poly(vinyl alcohol) (PVA) (Li \& Hsieh, 2006; Shan, Zhi, \& Jun, 2007). Electrospinning of Cs has been investigated by various authors all of which conclude that the production of pure Cs nanofibers was very challenging (Duan, Dong, Yuan, \& Yao, 2004; Huang, Ge, \& Xu, 2007; Li \& Hsieh, 2006). However, some authors reported success in electrospinning homogeneous Cs nanofibers from Cs solutions using only concentrated aqueous acetic acid (Geng, Kwon, \& Jang, 2005) or relatively high concentration and low molecular weight (Kriegel et al., 2009a). 
The electrospinning process can be manipulated by a number of variables. Quynh et al. (2006) reported that parameters that control the process can be classified in terms of solution properties, controlled variables, and ambient parameters. Solution properties involved surface tension, viscosity, conductivity, polymer molecular weight, and dielectric constant. The effects of the solution properties can be difficult to control since varying one parameter can generally affect other solution properties (e.g., changing the molecular weight can also change the viscosity). Controlled variables include the flow rate, electric field strength, distance between tip and collector, needle tip design, and collector composition and geometry. Ambient parameters include temperature, humidity, and air velocity.

In this study, nanofibers were manufactured by electrospinning solutions of a blend containing PEO with two different molecular weights of Cs. Three concentrations of aqueous acetic acid were used. The co-spinnig polymer PEO was chosen mostly for its excellent electrospinning characteristics to form utra-fine fibers, for its biocompatibility and for the fact that it is one of the few synthetic polymers approved for internal use in pharmaceutical, cosmetics, and food (Bhattarai et al., 2005). Limited research was conducted to improve the formation and the functionality of Cs nanofibers using surfactants. The addition of surfactants can be used to develop spinnability of solutions to yield natural nanofibers. Surfactants are amphiphilic molecules that readily absorb at surfaces and thereby lower the interfacial tension; a key parameter that influences electrospinning. Bhattarai et al. (2005) studied the use of Triton X-100 and Kriegel et al. (2009a) evaluated the effect of anionic sodium dodecyl sulfate (SDS), nonionic polyoxyethylene glycol lauryl ether (Brij 35), and cationic dodecyltrimethylammonium bromide (DTAB). It has been reported that the addition of a small amount of nonionic surfactant to polymer solutions reduced the onset voltage required to induce spinnability and improved reproducibility of the electrospinning process (Yao et al., 2003). Kriegel, Kit, McClements, and Weiss (2009b) found that in systems of nonionic polymers, nonionic surfactants did not stop bead formation but greatly reduced it. The main aim of the present study was to electrospin Cs nanofibers with two different molecular weights from a wide range of acetic acid concentrations. Crystallinity and the effect of acidity and molecular weight on solution properties and on the size and morphology of the nanofibrous structures generated were evaluated. The effect of nonionic surfactant Tween 20 on the electrospinning of the cationic biopolymer solution was also investigated.

\section{Materials and methods}

\subsection{Materials}

Two types of Cs were used; Cs (A) with molecular weight of $68 \mathrm{kDa}$ derived from cell fungi was obtained from Kitozyme (Batch L07319CsU, Herstal, Belgium). Cs (B) derived from shrimp shells was obtained from Primex (Reykjavik, Iceland), as stated by the manufacturer, the molecular weight was $\mathrm{Mw}=148 \mathrm{kDa}$. The degree of deacetylation of both was around 90\%. PEO (Cat \#343) with a molecular weight of $900 \mathrm{kDa}$ was purchased from Aldrich, USA. Glacial acetic acid was purchased from Mobi-Lab \& Labotec. Tween 20 (polyoxyethylene sorbitan monolaurate) (\#71729) was obtained from Fluka. All reagents were used as received from the manufacturer without further purification.

\subsection{Methods}

\subsubsection{Preparation of solutions}

All solutions were prepared from deionized water. Pure acetic acid was mixed with water to obtain concentrations of $10 \%, 50 \%$ and $90 \%(\mathrm{v} / \mathrm{v}) .5 \%(\mathrm{w} / \mathrm{v})$ of Cs was dissolved in acetic acid solution. To prepare blend solutions (Cs-PEO), $1 \mathrm{~g}$ of PEO solution in water $(4 \%(\mathrm{w} / \mathrm{v}))$ was added to $5 \mathrm{ml}$ of the different Cs solutions before spinning. For the study of the surfactant effect, $40 \mathrm{mg}$ of Tween 20 (above the critical micellar concentration) was incorporated to the spinnable solution. All solutions were kept under constant stirring overnight to ensure complete dissolution of the polymers and to obtain homogeneous solutions.

\subsubsection{Crystallinity}

The X-ray diffraction (XRD) patterns of polymers and the electrospun Cs nanofibers were recorded at ambient temperature on an X-ray diffractometer (Siemens D5000, Germany). Samples were dried for $24 \mathrm{~h}$ in the $0 \% \mathrm{RH}$ desiccator. After, they were analyzed between $5^{\circ}$ and $40^{\circ}(2 \theta)$ with monochromatized $\mathrm{Cu} \alpha(1.5405 \AA)$ $\mathrm{X}$-ray source. The operating voltage and current used were $40 \mathrm{kV}$ and $40 \mathrm{~mA}$, respectively.

\subsubsection{Viscosity of solutions}

The equipment employed to obtain rheograms consisted of a coaxial cylinder viscometer Haake Rotovisco RV 1 (Mess-Technik GmbH U. Co., Germany) equipped with a sensor system Z 20 DIN $\mathrm{Ti}$, in combination with a Haake DC30-K15 refrigerated circulator. Solutions were equilibrated to $25^{\circ} \mathrm{C}$ prior to all measurements and non linear regression was employed to fit experimental data to power law model [Eq. (1)].

$\tau=k \gamma^{n}$

That calculation was carried out by means of RheoWin Pro 3.40 software. Several variables were employed in order to find out if there is significant differences among brands. Consistency index $(K)$ and flow behavior index $(n)$ were directly obtained from the power law model whereas apparent viscosity at $100 \mathrm{~s}^{-1}$ (visc100) was calculated from the constant rate curves as the average of the last $50 \mathrm{~s}$ of measurement.

\subsubsection{Surface tension analysis}

The ring method with a tensiometer K9 (Krüss, Germany) was used to determine surface tension of the solutions. All measurements were made at $20^{\circ} \mathrm{C}$. Three replications were performed in all cases.

\subsubsection{Electrospinning of solutions}

In a $5 \mathrm{ml}$ syringe (Codan Medical, Rodby, Denmark), $2 \mathrm{ml}$ of polymer dispersions were placed. The syringe was placed in a syringe pump (Bioblock Scientific, Model A-99; Paris, France) which permitted adjustment and control of solution flow rates. The metal capillary of the syringe was connected to the positive lead of a high voltage power supply (Gamma High Voltage, ES 30P-5W; Ormond Beach, FL, USA), operated in positive DC mode that could generate voltages up to $30 \mathrm{kV}$. A grounded copper plate wrapped in aluminum foil and mounted onto two polypropylene blocks was used as the target collector plate for collection of fibers and/or beads. The target was placed $20 \mathrm{~cm}$ from the capillary tip. The syringe pump delivered polymer solution at a controlled flow rate of $0.03 \mathrm{ml} / \mathrm{min}$, while the voltage was maintained at $25 \mathrm{kV}$ and the temperature at $24{ }^{\circ} \mathrm{C}$. These conditions were kept constant throughout all experiments.

\subsubsection{Scanning electron microscopy}

The morphology of electrospun nanofibers was observed with a field emission scanning electron microscope FEG-ESEM XL 30 from FEI operated at $10 \mathrm{kV}$ in high vacuum conditions. Nanofibers were electrospun directly onto aluminum SEM stubs which were mounted on the grounded collector plate. After collection of the fibers, samples were sputter coated with gold in a Bal-Tec SCD 004 


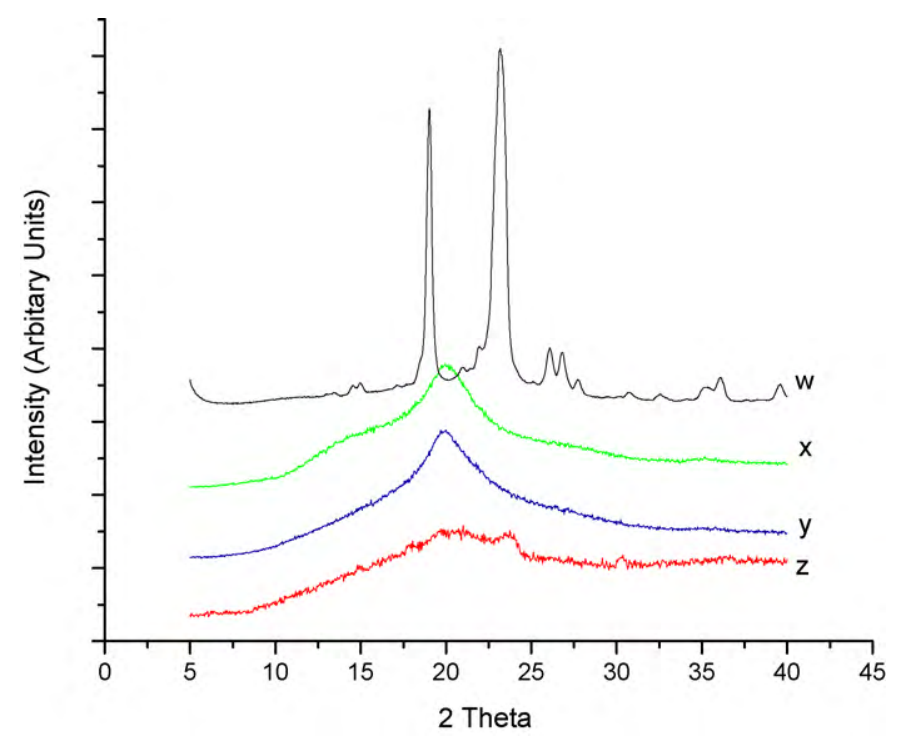

Fig. 1. XRD patterns of $(w)$ pure PEO, $(x)$ pure chitosan powder (148 kDa). (y) Pure chitosan (69 kDa). (z) Membrane of electrospun chitosan 148 kDa PEO + Tween 20.

plasma sputter coater for $40 \mathrm{~s}$. The average fiber diameter analysis was performed using image analysis software (Image J, NIH, USA) from $>50$ randomly selected fibers for each sample.

\subsubsection{Statistical analysis}

SPSS (Statistical Product and Service Solutions) version 12.0 was used for all statistical analyses. Analyses of variance (ANOVA) and Duncan means comparison test with a significance level of 0.05 were applied.

\section{Results and discussion}

\subsection{Crystallinity}

The main parameters that determine properties of Cs are molecular weight, deacetylation degree and polymer crystallinity. Fig. 1 shows the diffraction patterns of pure PEO, pure Cs (A and B), and their blends in fibrous form from electrospinning. No important differences were observed between both Cs although there was difference in source of the chitin. Only a broad band is clearly visible for both (Fig. $1 \mathrm{x}$ and y), whose maximum occurs around $2 \theta=20^{\circ}$ associated with the crystalline regions of the linear Cs molecules (Zhang, Su, Ramakrishna, \& Lim, 2008). Very slight shoulder at $2 \theta=12.5$ which can be attributed to the anhydrous crystal of Cs (B). Pure PEO shows very strong reflections at $19^{\circ} 2 \theta$ and $23^{\circ} 2 \theta$ and some

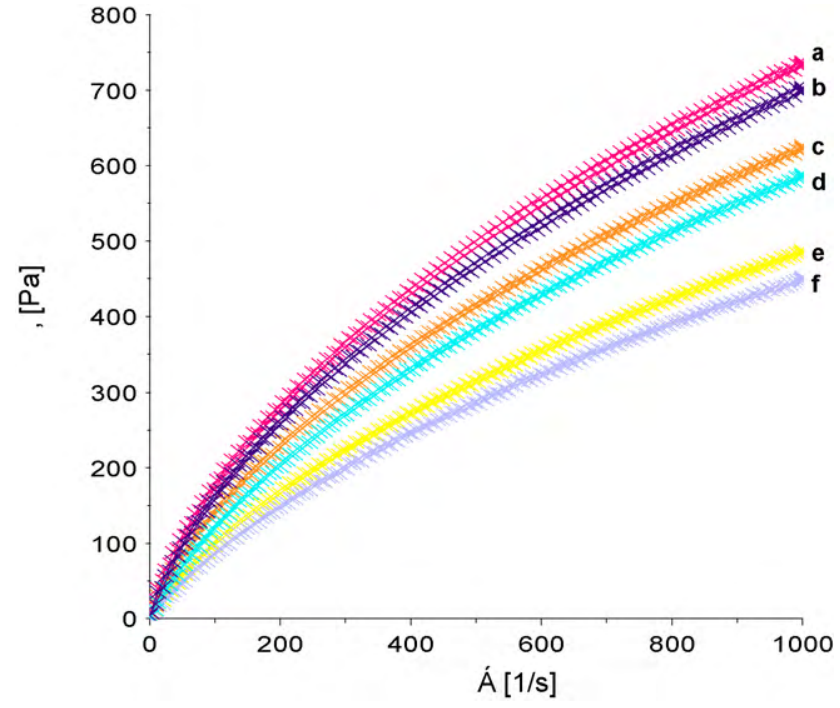

Fig. 2. Shear stress curves of $148 \mathrm{kDa}$ chitosan solutions. (a) In $90 \%$ of $\mathrm{Aa}$, (b) in $90 \%$ Aa with Tween 20 , (c) in $50 \% \mathrm{Aa}$, (d) in $50 \%$ Aa with Tween 20 , (e) in $10 \% \mathrm{Aa}$, (f) in 10\% Aa with Tween 20 .

weak reflections indicating its overall higher degree of crystallinity (Fig. 1w).

After electrospinning, the blended fibers (PEO-Cs, Fig. 1z) exhibit a broad peak around $20^{\circ} 2 \theta$ and a weak reflection around $24^{\circ} 2 \theta$. The low overall diffracted intensity in the blend. Neither the PEO nor the Cs retains its typical diffraction peaks, indicating a mutual interference effect in their respective crystallization process. This has been attributed to the formation of strong hydrogen bonding between ether groups from PEO and amino groups from Cs (Zhang et al., 2008). Electrospinning is supposed to delay the crystallization of polymers (Zhang et al., 2009), due to the stretching of macromolecules that rapidly solidify with high elongation rates. This significantly hinders the formation of crystals that normally occur by slow folding of polymer chains.

\subsection{Rheological properties}

The solution viscosity is a critical factor that affects solution spinnability and morphology of as-spun fibers. Viscosity is directly related to concentration, molecular weight, and the structure of the polymer as well as solvent type (Kriegel, Kit, McClements, \& Weiss, 2009c; Ramakrishna, 2005). Therefore, the rheological behavior of the polymer solution for any given sample is important. Fig. 2 shows shear stress curves of Cs B (148 kDa) as a function of the shear rate.

Table 1

Composition, power law flow behavior index $(n)$, consistency coefficient $K$ and viscosity of solutions.

\begin{tabular}{|c|c|c|c|c|c|c|c|}
\hline Solutions & Mw Cs (kDa) $)^{a}$ & Acetic acid $(\%, v / v)$ & $\begin{array}{l}\text { PEO } \\
\text { Yes }(\mathrm{Y}) / \text { no }(\mathrm{N})\end{array}$ & $\begin{array}{l}\text { Tween } 20 \\
\text { Yes }(\mathrm{Y}) / \text { no }(\mathrm{N})\end{array}$ & K & $n$ & Viscosity at $100 \mathrm{~s}^{-1}$ (Pas) \\
\hline 1 & - & - & $\mathrm{Y}$ & $\mathrm{N}$ & 9.41 & 0.49 & 0.874 \\
\hline 2 & 148 & 10 & $\mathrm{~N}$ & $\mathrm{~N}$ & 5.55 & 0.67 & 1.114 \\
\hline 3 & 148 & 50 & $\mathrm{~N}$ & $\mathrm{~N}$ & 7.51 & 0.66 & 1.405 \\
\hline 4 & 148 & 90 & $\mathrm{~N}$ & $\mathrm{~N}$ & 7.35 & 0.66 & 1.407 \\
\hline 5 & 148 & 10 & Y & $\mathrm{N}$ & 5.34 & 0.66 & 1.002 \\
\hline 6 & 148 & 50 & $\mathrm{Y}$ & $\mathrm{N}$ & 8.69 & 0.62 & 1.411 \\
\hline 7 & 148 & 90 & Y & $\mathrm{N}$ & 11.89 & 0.60 & 1.773 \\
\hline 8 & 148 & 10 & $\mathrm{Y}$ & $\mathrm{Y}$ & 4.11 & 0.68 & 0.852 \\
\hline 9 & 148 & 50 & $\mathrm{Y}$ & $\mathrm{Y}$ & 6.48 & 0.65 & 1.214 \\
\hline 10 & 148 & 90 & $\mathrm{Y}$ & $\mathrm{Y}$ & 10.17 & 0.62 & 1.616 \\
\hline 11 & 68 & 10 & Y & $\mathrm{Y}$ & 4.56 & 0.69 & 0.984 \\
\hline 12 & 68 & 50 & $\mathrm{Y}$ & $\mathrm{Y}$ & 7.03 & 0.66 & 1.321 \\
\hline 13 & 68 & 90 & $\mathrm{Y}$ & $\mathrm{Y}$ & 10.51 & 0.62 & 1.720 \\
\hline
\end{tabular}

\footnotetext{
a Concentration of chitosan $5 \%$.
} 


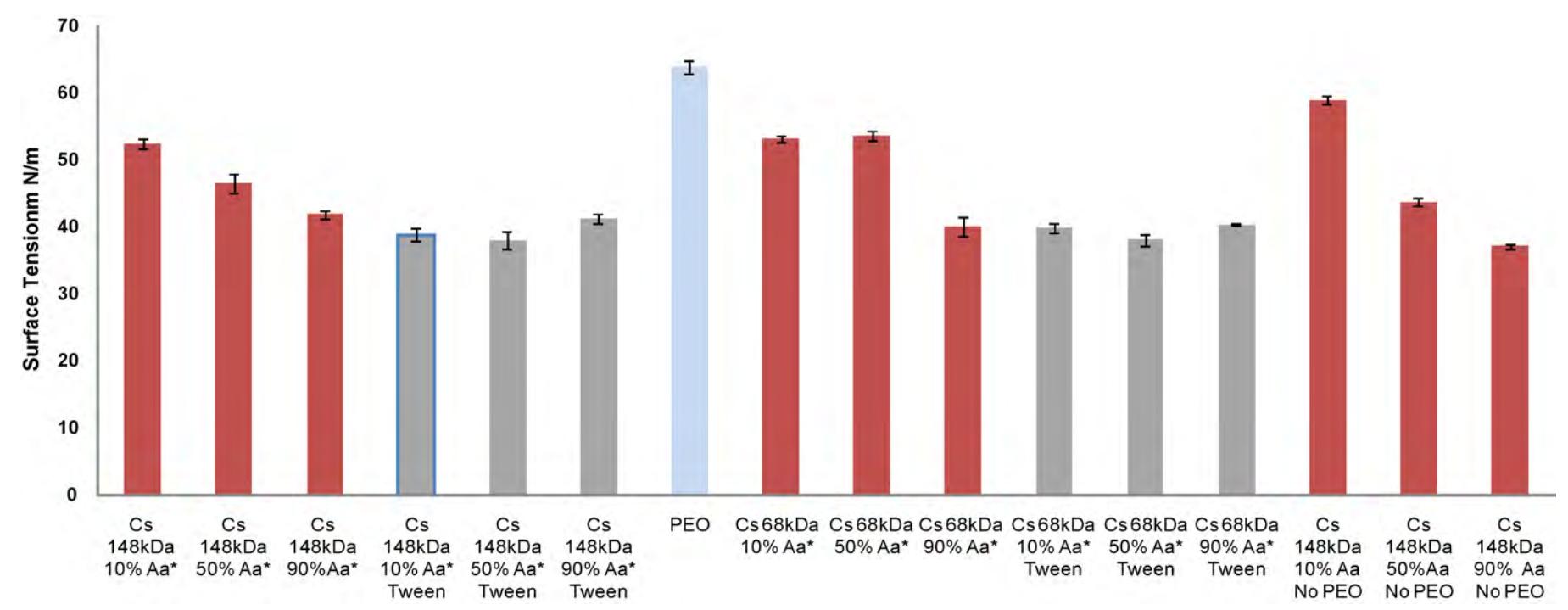

Fig. 3. Surface tension of solutions ( ${ }^{*}$ with PEO; $\mathrm{Aa}=$ acetic acid).

It was observed that viscosity range of different spinnable solutions was different. The effect of acidity and the use of Tween 20 were observed. Solutions with high acidity were more viscous and for those including Tween 20 in their formulation a little effect was observed. The viscosity decreases in all solutions when Tween 20 was added. This decrease was more effective for Cs solution with the high molecular weight (Table 1 ). To gain a more in-depth understanding of the rheological behavior of solutions, apparent viscosity, the consistency coefficient $K$ and flow behavior index $n$ at a shear rate of $100 \mathrm{~s}^{-1}$ were also evaluated. Table 1 shows that apparent viscosities of all solutions with PEO and Cs dissolved at the highest acetic acid concentration 90\% represented the highest value of viscosity (higher than 1.6 Pa s). The addition of surfactant had an effect on solution viscosity, e.g. addition of Tween 20 to all Cs (148 kDa) solutions with PEO at the respective concentration (10\%, $50 \%$ and $90 \%$ ) of acetic acid reduces the apparent viscosity from $1.00,1.41,1.77$ Pa s to $0.85,1.21$ and 1.61 Pa s, respectively.

The PEO solution had a lowest viscosity value ( $0.87 \mathrm{~Pa}$ s) at shear rate of $100 \mathrm{~s}^{-1}$. It can be seen that the effect of PEO on Cs solution viscosity depends on the acidity level. For solution in $10 \%$ of acetic acid, the addition of PEO to Cs leads to a decrease in viscosity from 1.114 to $1.002 \mathrm{~Pa}$ s. On the opposite, PEO addition to solution in high acidity (50\% and $90 \%$ of acetic acid) leads to an increase of the viscosity. The same result was reported in Kriegel et al. (2009a) work on the addition of PEO to Cs solutions with $50 \%$ and $90 \%$ of acetic acid.

The flow behavior index $(n)$ is an indicator of whether the solution has a tendency to behave like a shear thinning, shear thickening or Newtonian liquid. All polymer solutions regardless of composition had indices smaller than 1 suggesting a pseudoplastic behavior. PEO solution exhibits a low value results of $n(n=0.49)$, while other solutions present a value around 0.6 . The increase of acidity in all solutions results in solutions with smaller flow behavior index. With the addition of the nonionic surfactant the flow behavior index increased slightly: e.g. for Cs ( $148 \mathrm{kDa}$ ) solutions at 10\%, 50\% and $90 \%$ of acetic acid the $n$ index increases from $0.66,0.62,0.60$ to $0.68,0.65,0.62$, respectively. A reduced $(n)$ is generally an indication of polymer entanglement, which is a fundamental prerequisite for deposition of nanofibers during the electrospinning process.

The consistency coefficient provides a measure of the apparent viscosity of the solution at low shear rates. The higher $K$ results in more viscous solution. The consistency coefficient $K$ followed similar trends as the apparent viscosity discussed above. $K$ values increased generally with the increase of acetic acid rate. In the pres- ence of Tween 20 it was observed that the consistency coefficient decreased. In Kriegel et al. (2009a) work, the effect on consistency coefficient depends on the nature of used surfactant (nonionic, cationic etc).

The observed rheological behavior of polymer solutions in the presence of surfactant suggests that polymer-polymer interactions are modulated by the presence of surfactant and the concentration of the acid in which the polymers are dissolved. For the use of different rates of acetic acid, the increasing of acidity for both high degree of deacetylation Cs used in this work leeds to an increase of the amount of charge of free amine on the chain. As the charge on the chain increases, the conformation of the chain in solution expands and the viscosity increases substantially (Klossner, Queen, Coughlin, \& Krause, 2008). For the use of surfactant, Tween 20 as a nonionic surfactant would be expected to show no electrostatic interaction and instead may interact with polymers solely by hydrophobic bonding. The fact that addition of Tween 20 to the polymer solutions caused little change in viscosity suggests that it did not strongly affect the interactions between the polymer molecules.Surface tension

ST of a solution is often defined as the force acting at right angles to any line of unit length on the liquid surface (Haghi \& Akbari, 2007). For electrospinning, the jet is initiated at the point where the electrostatic force in solution overcomes the ST of the solution (Homayoni, Ravandi, \& Valizadeh, 2009). In general, ST determines the upper and lower limits of electrospinning window if all other variables are held constant. The formation of droplets, bead and fibers can be driven by the ST of Cs solution. Lower ST of the spinning solution helps electrospinning to occur at lower electric field (Geng et al., 2005; Homayoni et al., 2009). Fig. 3 represents the surface tension of various solutions used in this study. It is observed that the PEO solution shows a highest ST value $(63.9 \pm 0.9 \mathrm{mN} / \mathrm{m})$ while the lowest value $(37.1 \pm 0.3 \mathrm{mN} / \mathrm{m})$ was for solution with Cs $148 \mathrm{kDa}$ (without added surfactant and PEO) at $90 \%$ of acetic acid. As for viscosity, it is observed that the effect of PEO on Cs solution ST depends on the acidity. The addition of the neutral polymer PEO to Cs solution leads to a decrease in ST for Cs ( $148 \mathrm{kDa})$ solution with acidity at $10 \%$ and an increase for those in $50 \%$ and $90 \%$ of acetic acid.

It is observed that the increase of acetic acid from $10 \%$ to $90 \%$ leads to the decrease of the ST of both Cs (68 kDa and $148 \mathrm{kDa}$ ) solutions without added surfactant. The use of the nonionic surfactant (Tween 20) in solution also reduced significantly the ST of both Cs solutions dissolved at $10 \%$ and $50 \%$, i.e. from 52.4 and $46.4 \mathrm{mN} / \mathrm{m}$ 

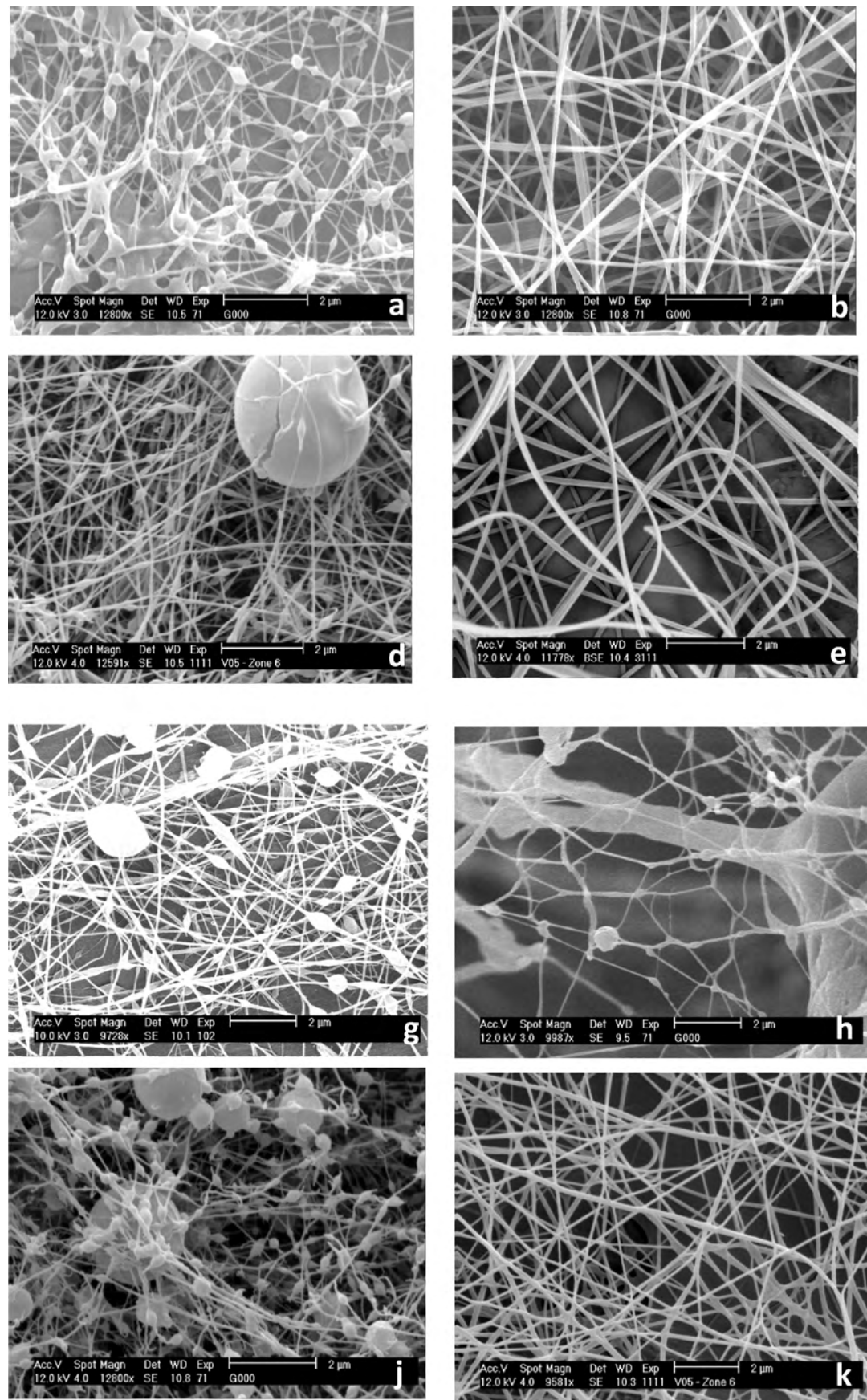
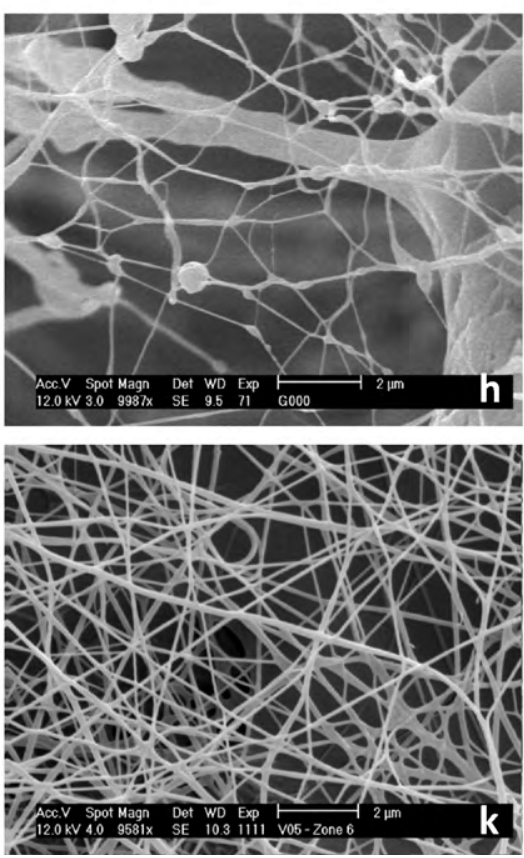
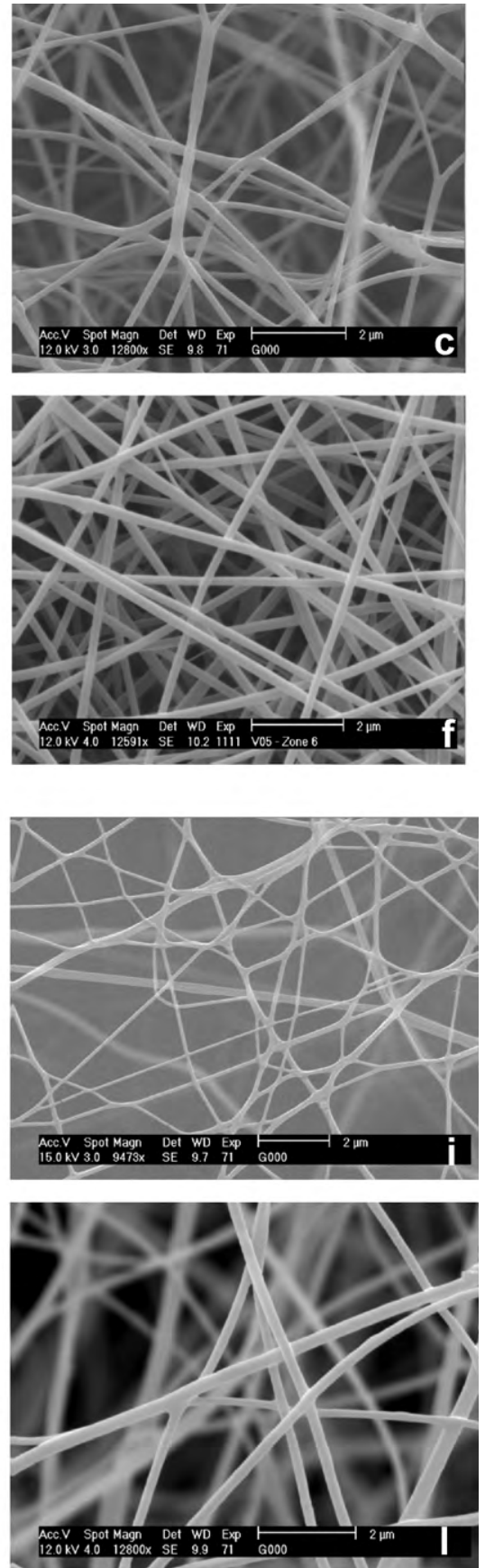

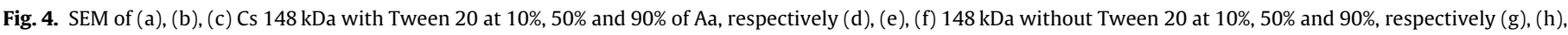
(i) Cs $68 \mathrm{kDa}$ with Tween 20 at 10\%, 50\% and 90\% of Aa, respectively (j), (k), (l) $68 \mathrm{kDa}$ without Tween 20 at $10 \%, 50 \%$ and $90 \%$.

for solutions of $148 \mathrm{kDa}$ with PEO to 38.8 and $38 \mathrm{mN} / \mathrm{m}$ for same solution including the surfactant. For solutions with $90 \%$ of acetic acid the addition of the surfactant did not have a significant effect on ST. No synergic effect was observed.The importance of the effect of acidity on ST can be associated with the net charge density of the Cs solution that increased with increasing acetic acid concentration in water resulting in more charged ions available for charge repulsion.

For the addition of the nonionic surfactant; Tween 20 exhibits low affinity for polyelectrolytes as associations are solely based on hydrogen bonding and/or hydrophobic interactions. This explains the more pronounced effect that Tween 20 had both solution viscosity and ST.In solutions where the only difference was the molecular weight, no significant difference was observed on ST between the Cs (A) and Cs (B). ST of Cs solutions was related to the conformation (Qun \& Ajun, 2006).The effect of molecular weight on the conformation of Cs molecules in dilute solution was studied by Tsaih and Chen (1997). They found that conformation of Cs whose molecular weights were higher than or equal to $223 \mathrm{kDa}$ were in a random coil, whereas for Cs whose molecular weights were lower or equal to $148 \mathrm{kDa}$ were in a rod shape. They suggested that the occurrence of molecular weight induced conformational transition of Cs might be attributed to differences in intra-molecular hydrogen bonds and/or difference in charge distribution among larger and smaller molecular weight Cs. The ST of Cs solutions are mainly determined by hydrogen bonding, hydrophobic interaction and electrostatic interaction. There is a plenty of $\mathrm{OH}$ and $\mathrm{NH}_{2}$ groups on the Cs molecular chains, a strong hydrogen bonding 


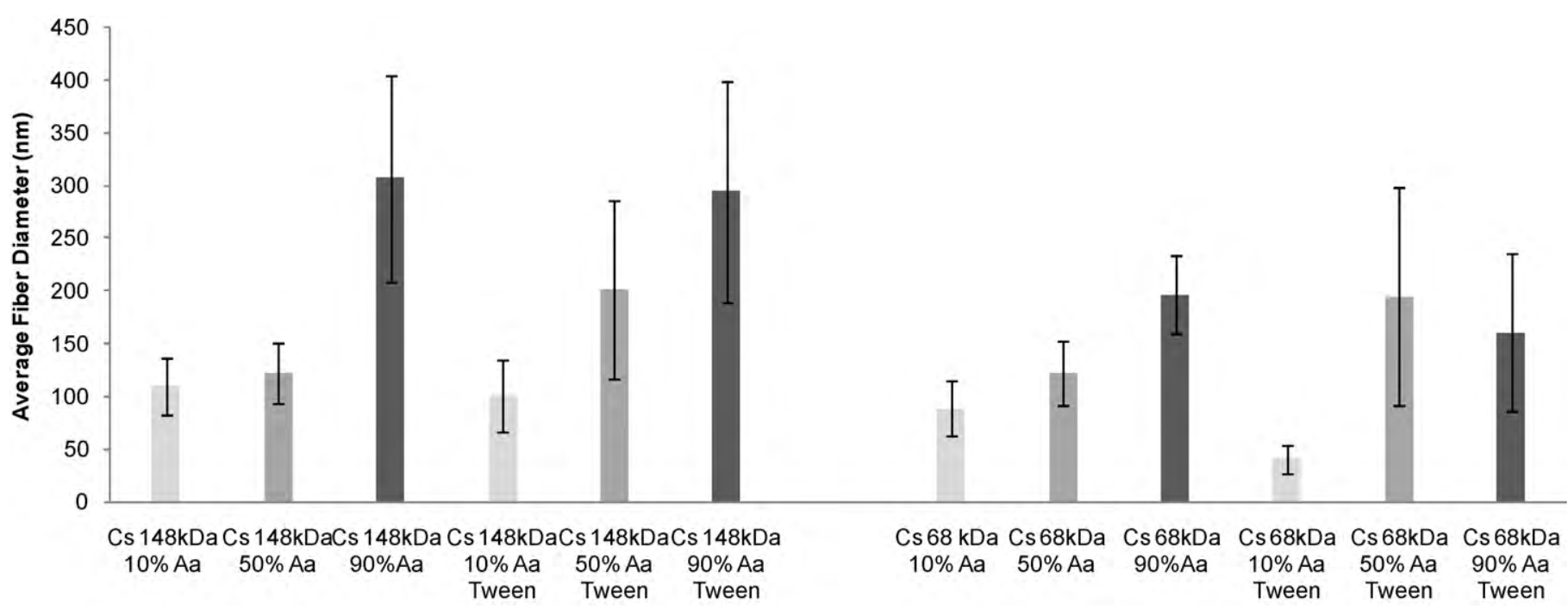

Fig. 5. Average diameters of chitosan nanofibers.

and electrostatic interaction may exist in acid aqueous solution. For different molecular weight Cs solutions, based on the Tsaih's study mentioned above, the molecules of high molecular weight Cs entangle easier than low molecular weight ones, and therefore, the high molecular weight Cs should have more inter-molecular hydrogen bonding. Consequently, the surface tension of high molecular weight Cs is larger than the low molecular weight ones. In our case, we suggest that the same degree of deacetylation of the Cs used had more effect on ST than the range of molecular weight (Cs (A) and (B)). For the same degree of deacetylation Cs in solution with the same ionic strength and $\mathrm{pH}$, the degree of protonation should be the same; the hydrogen bonding interaction will dominate the surface tension changes (Qun \& Ajun, 2006) and consequently may give same ST.Morphology and fiber diameter

The electrospinning of Cs and other natural polymers is a complex process because of the special behavior of the solution of this polymer, its polycationic specification, its high molecular weight, and its wide molecular weight distribution (Homayoni et al., 2009). Therefore, the morphology of electrospun fibers depends mainly on the interaction between the different properties of Cs electrospun solution.

During the electrospinning process, it was observed that when using a high acidity (90\%) the jet was directed towards a centered zone which permits the formation of a cone with multilayer of nanofibers on the collector plate. On the contrary, the electrospinning of solutions in $10 \%$ of acetic acid resulted in the dispersion of jet forming an extended membrane on the collector surface. This can be explained by the fact that at low acidity, chain entanglement may not be sufficient, resulting in spraying of large beads connected by very thin fibers.

Fig. 4 shows the representative images of all studied electrospun nanofibers. It was observed that all images resulting from solutions at the highest acidity (Fig. 4c, i, f, l) present less beads and beaded fibers than the others. This is related to the solutions properties studied above and to the increase of acetic acid concentration that gave viscous solutions with lower surface tension and an adequate polymer chain entanglement.

In this work, the molecular weight range of both Cs and the selected concentration permit the electrospinning of good nanofibers. The loss of electrospinnability in solutions is often due to reduced or insufficient polymer chain entanglement required for the formation of a stable and continuous polymer jet that has to be ejected from the tip of the capillary (Buchko, Chen, Shen, \& Martin,
1999). Insufficient entanglement can lead to a break up of the jet into small droplets, yielding beads instead of fibers.

The average diameters of fibers was determined from image analysis (Fig. 5). The highest Cs molecular weight without Tween at $90 \%$ of acetic acid gave fibers with the highest average diameter $(307 \mathrm{~nm})$. The most significant factor for the fiber's diameter was the acidity level since an increase in the amount of acetic acid led to an increase of the average fiber diameter. Solutions in $90 \%$ of Aa gave fiber diameters around $300 \mathrm{~nm}$ for Cs B and around $190 \mathrm{~nm}$ from Cs A solutions. For Cs solution in $10 \%$ acetic acid, the average diameter was around $105 \mathrm{~nm}$ for Cs B with or without Tween 20 and $88 \mathrm{~nm}$ and $40 \mathrm{~nm}$ for Cs A with Tween 20 and without it, respectively.

The highest molecular weight Cs (B) gave a larger average diameter but without a significant difference with those electrospun from Cs (A). Results reported previously (Deitzel, Kleinmeyer, Harris, \& Beck Tan, 2001; Geng et al., 2005; Koski, Yim, \& Shivkumar, 2004) showed that very low molecular weight polymers have a tendency to form beads rather than fibers and high molecular weight polymers give fibers with larger average diameter if the concentration of the polymer is adequate. For the effect of Tween 20, it was neither a significant factor on fiber diameter, at the contrary in Shu-Qiang, Ji-Huan, and Lan (2008) work, the average diameter of the fibers decreased as the concentration of the nonionic Triton $\mathrm{X}-100$ increased.

\section{Conclusions}

The chain entanglement is remarkably important in Cs electrospinning. The electrospinning of Cs/acetic acid system showed that acetic acid concentration strongly influenced the chain entanglement of the Cs and consequently the properties of Cs solutions and the morphology of the formed fibers. The chain entanglement can depend on molecular weight but not on all ranges as showed in this study.

By reducing surface tension of solution, fibers could be obtained without beads. Different solvents may contribute different surface tensions; however, lower surface tension of a solvent may not necessarily be more suitable for electrospinning.

The addition of decreasing ST agent (surfactant) did not improve the quality of nanofibers but the fact that surfactant was cospun with Cs could make it possible for micelles, this could serve as an additional means to further functionalize fibers and 
broaden the number of applications in which nanofibers could be used.

\section{Acknowledgments}

This research was supported by funding from GoCell project. The authors would like to thank Wallonia region in Belgium for its financial support.

\section{References}

Bhattarai, N., Edmondson, D., Veiseh, O., Matsen, F. A., \& Zhang, M. (2005). Electrospun chitosan-based nanofibers and their cellular compatibility. Biomaterials, 26, 6176-6184.

Buchko, C. J., Chen, L. C., Shen, Y., \& Martin, D. (1999). Processing and microstructural characterization of porous biocompatible protein polymer thin films. Polymer, 40, 7397-7407.

Deitzel, J. M., Kleinmeyer, J., Harris, D., \& Beck Tan, N. C. (2001). The effect of processing variables on the morphology of electrospun nanofibers and textiles. Polymer, $42,261-272$.

Duan, B., Dong, C., Yuan, X., \& Yao, K. (2004). Electrospinning of chitosan solutions in acetic acid with poly(ethylene oxide). Journal of Biomaterials Science, 15, 797-811.

Du, J., \& Hsieh, Y. L. (2007). PEGylation of chitosan for improved solubility and fiber formation via electrospinning. Cellulose, 14, 543-552.

Geng, X., Kwon, O., \& Jang, J. (2005). Electrospinning of chitosan dissolved in concentrated acetic acid solution. Biomaterials, 26, 5427-5432.

Haghi, A. K., \& Akbari, M. (2007). Trends in electrospinning of natural nanofibers. Physica Status Solidi, 204, 1830-1834.

Homayoni, H., Ravandi, S., \& Valizadeh, M. (2009). Electrospinning of chitosan nanofibers: Processing optimization. Carbohydrate Polymers, 7, 656661.

Huang, X. J., Ge, D., \& Xu, Z. K. (2007). Preparation and characterization of stable chitosan nanofibrous membrane for lipase immobilization. European Polymer Journal, 43(9), 3710-3718.

Kafetzopoulos, D., Martinou, A., \& Bouriotis, V. (1993). Bioconversion of chitin to chitosan: Purification and characterization of chitin deacetylase from Mucor rouxii. Proceedings of the National Academy of Science, 90, 2564-2568.

Klossner, R. R., Queen, H. A., Coughlin, A. J., \& Krause, W. E. (2008). Correlation of chitosan's rheological properties and its ability to electrospin. Biomacromolecules, 9, 2947-2953.
Koski, A., Yim, K., \& Shivkumar, S. (2004). Effect of molecular weight on fibrous PVA produced by electrospinning. Materials Letters, 58, 493-497.

Kriegel, C., Kit, K. M., McClements, D. J., \& Weiss, J. (2009a). Electrospinning of chitosan-poly(ethylene oxide) blend nanofibers in the presence of micellar surfactant solutions. Polymer, 50, 189-200.

Kriegel, C., Kit, K. M., McClements, D. J., \& Weiss, J. (2009b). Influence of surfactant type and concentration on electrospinning of chitosan-poly(ethylene oxide) blend nanofibers. Food Biophysics, 4, 213-228.

Kriegel, C., Kit, K. M., McClements, J., \& Weiss, J. (2009c). Nanofibers as carrier systems for antimicrobial microemulsions. Part I: Fabrication and Characterization. Langmuir, 25, 1154-1161.

Li, L., \& Hsieh, Y. L. (2006). Chitosan bicomponent nanofibers and nanoporous fibers. Carbohydrate Research, 341, 374-381.

Qun, G., \& Ajun, W. (2006). Effects of molecular weight, degree of acetylation and ionic strength on surface tension of chitosan in dilute solution. Carbohydrate Polymers, 64, 29-36.

Quynh, P., Sharma, U., \& Mikos, A. G. (2006). Electrospinning of polymeric nanofibers for tissue engineering applications. Tissue Engineering, 12, 1197-1211.

Ramakrishna, S. (2005). An introduction to electrospinning and nanofibers. Singapore: World Scientific.

Shalumon, K. T., Binulal, N. S., Selvamurugan, N., Nair, S. V., Menon, D., Furuike, T., et al. (2009). Electrospinning of carboxymethyl chitin/poly(vinyl alcohol) nanofibrous scaffolds for tissue engineering applications. Carbohydrate Polymers, 77, 863-869.

Shan, Z.Y.,Zhi, Y.D., \& Jun, N. (2007). Preparation and characterization of crosslinked chitosan-based nanofibers. Chinese Chemical Letters, 18, 118-120.

Shu-Qiang, W., Ji-Huan, H., \& Lan, X. (2008). Non-ionic surfactants for enhancing electrospinnability and for the preparation of electrospun nanofibers. Polymer International, 57, 1079-1082.

Tsaih, M. L., \& Chen, R. H. (1997). Effect of molecular weight and urea on the conformation of chitosan molecules in dilute solutions. International Journal of Biological Macromolecules, 20, 233-240.

Yao, L., Haas, T. W., Guiseppi-Elie, A., Bowlin, G. L., Simpson, D. G., \& Wnek, G. E. (2003). Electrospinning and stabilization of fully hydrolyzed poly(vinyl alcohol) fibers. Chemistry of Materials, 15(9), 1860-1864.

Zentz, F., Bédouet, L., Almeida, M. J., Milet, C., Lopez, E., \& Giraud, M. (2001). Characterization and quantification of chitosan extracted from nacre of the abalone haliotis tuberculata and the oyster pinctada maxima. Marine Biotechnology, 3, 36-44.

Zhang, H., Li, S., Branford White, C. J., Ning, X., Nie, H., \& Zhu, L. (2009). Studies on electrospun nylon-6/chitosan complex nanofiber interactions. Electrochimica Acta, 54, 5739-5745.

Zhang, Y. Z., Su, B., Ramakrishna, S., \& Lim, C. T. (2008). Chitosan nanofibers from an easily electrospinnable UHMWPEO-doped chitosan solution system. Biomacromolecules, 9, 136-141. 